Article

\title{
Quantum Optimal Control of Rovibrational Excitations of a Diatomic Alkali Halide: One-Photon vs. Two-Photon Processes
}

\author{
Yuzuru Kurosaki ${ }^{1, *}$ and Keiichi Yokoyama ${ }^{2}$ \\ 1 Tokai Quantum Beam Science Center, Takasaki Advanced Radiation Research Institute, National Institutes \\ for Quantum and Radiological Science and Technology, Tokai, Ibaraki 319-1195, Japan \\ 2 Materials Science Research Center, Japan Atomic Energy Agency, Kouto, Sayo, Hyogo 679-5148, Japan; \\ yokoyama.keiichi@jaea.go.jp \\ * Correspondence: kurosaki.yuzuru@qst.go.jp
}

Received: 29 March 2019; Accepted: 3 May 2019; Published: 8 May 2019

\begin{abstract}
We investigated the roles of one-photon and two-photon processes in the laser-controlled rovibrational transitions of the diatomic alkali halide, ${ }^{7} \mathrm{Li}^{37} \mathrm{Cl}$. Optimal control theory calculations were carried out using the Hamiltonian, including both the one-photon and two-photon field-molecule interaction terms. Time-dependent wave packet propagation was performed with both the radial and angular motions being treated quantum mechanically. The targeted processes were pure rotational and vibrational-rotational excitations: $(v=0, J=0) \rightarrow(v=0, J=2) ;(v=0, J=0) \rightarrow(v=1, J=2)$. Total time of the control pulse was set to 2,000,000 atomic units (48.4 ps). In each control excitation process, weak and strong optimal fields were obtained by means of giving weak and strong field amplitudes, respectively, to the initial guess for the optimal field. It was found that when the field is weak, the control mechanism is dominated exclusively by a one-photon process, as expected, in both the targeted processes. When the field is strong, we obtained two kinds of optimal fields, one causing two-photon absorption and the other causing a Raman process. It was revealed, however, that the mechanisms for strong fields are not simply characterized by one process but rather by multiple one- and two-photon processes. It was also found that in the rotational excitation, $(v=0$, $J=0) \rightarrow(v=0, J=2)$, the roles of one- and two-photon processes are relatively distinct but in the vibrational-rotational excitation, $(v=0, J=0) \rightarrow(v=1, J=2)$, these roles are ambiguous and the cooperative effect associated with these two processes is quite large.
\end{abstract}

Keywords: quantum control; optimal control theory; diatomic molecule; two-photon absorption; Raman process

\section{Introduction}

Recent rapid developments in laser technology have prompted a huge number of studies aiming at a better understanding of very fast dynamical events behind physical, chemical, and biological phenomena. It is not until the developments of ultrashort pulse generation and the pulse shaping technique that one can use the quantum control idea [1-4] to achieve the goal of relevant physical processes. In recent years, quantum control has attracted great interest because of its potential for opening up a new frontier in fundamental science and has found vast application in chemical reaction [5,6], molecular alignment [7], quantum computation [8], laser cooling [9,10], etc.

In our view, the underlying mechanisms of quantum control may fall into three categories. The first one is the pump-dump scheme [5] often employed for chemical reactions hindered by a high energy barrier. The molecular system in the electronic ground state at first is electronically excited by a pump pulse and the nuclear wave packet forms and moves around on the excited potential surface. 
When large portions of the wave packet are localized in the region that is geometrically close to the target, the second dump pulse is applied to deexcite the wave packet to the ground state potential. The wave packet created again on the ground state potential may reach the target with high efficiency due to the geometrical vicinity between the wave packet and the target. The second category is the control scheme relying on interference arising via multiple pathways to the target [6]. One of the scenarios is the control of the product branching ratio in the photodissociation from a single state via two pathways: the $\mathrm{N}$-photon and M-photon optical routes. It was shown that the control parameters are the magnitudes of the N-photon and M-photon pulses and their phases and thus changing those values can make one dissociation channel predominate over the other. The last category is the scheme of stimulated Raman adiabatic passage (STIRAP) [11-13]. The basics of STIRAP are often described using the simplest three-state $\Lambda$ model; let states 1,2, and 3 be the initial, intermediate, and target states, respectively, and the objective is to transfer the population from state 1 to state 3 completely, induced by two coherent fields that couple states 2 to 1 and 3 (pump and Stokes pulses, respectively). When applying an appropriate electric field, the degeneracy of three eigenstates of the system Hamiltonian, which coincide with state 1 at first, is lifted and the three eigenstates temporally evolve adiabatically, and they finally become degenerate again and converge to state 3. It was shown that applying the Stokes pulse first and then the pump pulse, which is the so-called counterintuitive ordering, is needed to allow for the desired adiabatic evolution. Of particular note is that one of the eigenstates of the system Hamiltonian consists of states 1 and 3 and not of state 2, with the eigenvalue remaining at zero throughout the control process, as long as the two-photon resonance condition is met. Because of these basics, the STIRAP technique has been considered to realize efficient population transfer while minimizing loss due to spontaneous emission from state 2 . One of the most significant advances in STIRAP may be the technique referred to as "shortcut to adiabaticity" [14], which enables speeding up of the control time, and the derived protocol from it called the superadiabatic technique $[15,16]$ that suppresses the spurious nonadiabatic processes.

The idea of quantum optimal control can contain all the essences of the three categories described above. As a consequence, the control mechanisms obtained by optimal control theory (OCT) [17-19] and optimal control experiment (OCE) $[20,21]$ are sometimes hard to understand and the optimal control fields predicted by OCT are often hard to generate even with state-of-the-art pulse shaping technology; optimal control is, however, a very powerful and intriguing tool that can address various control problems for quantum systems.

We have been interested in the study of quantum control problems, focusing recently on applying OCT to isotope separation, which is an important subject to explore not only in basic science but in industrial applications. Using OCT, we [22-25] studied the isotope-selective vibrational excitations of diatomic molecules; the theoretical calculations predicted the control electric fields that can efficiently realize the isotope-selective excitations. We [26-29] also theoretically investigated pure rotational excitations of diatomic molecules using an optical frequency comb; note that in the current pulse shaping technology, the design of the frequency comb for rotational excitations of heavy diatomic molecules is more realistic than that of the excitation pulse for vibrational transitions. We [30] recently proposed a control scenario for the isotope-selective rovibrational excitation of diatomic molecules at a finite temperature; first a frequency comb for rotational excitations is applied to change the rotational-state distributions and to magnify the difference in vibrational transition energies between the isotopologues, and second, a bunch of pulse is irradiated for isotope-selective vibrational excitations. Thus it was shown in the numerical simulation that the proposed scheme works well for the isotope-selective rovibrational excitation for a gas-phase mixture of the diatomic alkali-halide ${ }^{7} \mathrm{Li}^{37} \mathrm{Cl}$ and ${ }^{7} \mathrm{Li}^{35} \mathrm{Cl}$ molecules at $70 \mathrm{~K}$.

In a previous study, we [31] performed OCT simulations for the isotope-selective rovibrational excitation of the diatomic alkali-halide isotopologues: ${ }^{7} \mathrm{Li}^{37} \mathrm{Cl}$ and ${ }^{7} \mathrm{Li}^{35} \mathrm{Cl}$, where both the radial (vibrational) and angular (rotational) motions were treated quantum mechanically in the wave-packet calculations. As a result, the calculated final yields were nearly 1.0, indicating that the optimal fields 
to achieve the goal were successfully obtained with the two-dimensional (2D) quantum mechanical method. In the previous study [31] however, only linear field effects were considered, i.e., the field-molecule Hamiltonian includes only the term linearly dependent on the applied field, which is responsible for one-photon processes. In the present study we added the term proportional to the square of the electric field, which is responsible for two-photon processes. To our knowledge, this is the first attempt to carry out 2D OCT simulations for diatomic molecules with the Hamiltonian including both the linear and nonlinear interaction terms. In this paper we consider only a single isotopic species, ${ }^{7} \mathrm{Li}^{37} \mathrm{Cl}$, because we intend to examine and compare the roles of one-photon and two-photon processes in rovibrational transitions, rather than the isotopic selectivity. Two control processes were investigated, i.e., the rotational excitation and vibrational-rotational excitation of the ${ }^{7} \mathrm{Li}^{37} \mathrm{Cl}$ molecule. The initial state is set to the vibrational and rotational ground levels, $v=0$ and $J=$ 0 , and the target states are the $v=0$ and $J=2$ state for the rotational excitation and the $v=1$ and $J$ $=2$ state for the vibrational-rotational excitation, i.e., we tried to theoretically control the following excitations: (i) $(v=0, J=0) \rightarrow(v=0, J=2)$; (ii) $(v=0, J=0) \rightarrow(v=1, J=2)$.

Theoretical details are described in Section 2. The results of calculations are presented in Section 3 and conclusions are given in Section 4.

\section{Theoretical Details}

\subsection{OCT}

In OCT calculations [17-19], an optimal electric field is found such that the objective functional $J$ is maximized, which is written as

$$
J=\langle\psi(T)|\hat{O}| \psi(T)\rangle-\int_{0}^{T} d t \frac{\varepsilon(t)^{2}}{\lambda}-2 \operatorname{Re}\left[\int_{0}^{T} d t\left\langle\chi(t)\left|\frac{\partial}{\partial t}+i \hat{H}(r, \theta, \varepsilon(t))\right| \psi(t)\right\rangle\right] .
$$

The first term is the transition probability to be maximized and is referred to as the final yield, where $\psi(T)$ is the system wave packet propagated from time $(t) 0$ to total time $T$ and $\hat{O}$ is the target operator. The second term is the laser fluence to be minimized, where $\varepsilon(t)$ is the electric field and $\lambda$ is a penalty factor. In order to obtain the optimal fields that start and end with zero amplitude, a time dependence is introduced in $\lambda$ :

$$
\lambda=\lambda(t)=\lambda_{0} s(t)
$$

where $\lambda_{0}$ is a positive constant and $s(t)$ is a shape function having a form

$$
s(t)=\sin ^{2}\left(\frac{\pi t}{T}\right)
$$

The third term is the dynamical constraint, where $\chi(t)$ is the Lagrange multiplier, Re denotes the real part of a complex number, $\hat{H}(r, \theta, \varepsilon(t))$ is the Hamiltonian depending on the internuclear distance $r$, the angle $\theta$ between the molecular axis and the laser polarization vector, and $\varepsilon(t)$.

Applying the variational approach to the functional $J$, coupled equations for $\psi(t), \chi(t)$, and $\varepsilon(t)$ are obtained and an optimal field $\varepsilon(t)$ is finally found by solving these equations iteratively until the value of $J$ is converged. We adopt a monotonically convergent algorithm $[32,33]$ that is capable of solving OCT problems in which a system interacts nonlinearly with an applied electric field. As, in this study, the nonlinear interaction is considered up to the term proportional to the square of the electric field $\varepsilon(t)$, it is artificially divided into two components $\varepsilon_{1}(t)$ and $\varepsilon_{2}(t)$ in the algorithm. Starting the iteration by solving $\psi^{(0)}(t)$ with a set of initial trial fields, $\varepsilon_{1}{ }^{(0)}(t)$ and $\varepsilon_{2}{ }^{(0)}(t)$, the $k$ th iteration step $(k \geq 1)$ according to the monotonically convergent algorithm is presented as follows:

$$
\frac{\partial}{\partial t} \chi^{(k)}(t)=-\left[\hat{H}_{0}+\frac{\hat{H}_{1}}{2}\left\{\varepsilon_{1}^{(k-1)}(t)+\varepsilon_{2}^{(k-1)}(t)\right\}+\hat{H}_{2} \varepsilon_{1}^{(k-1)}(t) \varepsilon_{2}^{(k-1)}(t)\right] \chi^{(k)}(t)
$$


with the final condition, $\chi^{(k)}(T)=\hat{O} \psi^{(k)}(T)$, and then $\varepsilon_{1}{ }^{(k)}(t)$ is updated as

$$
\varepsilon_{1}^{(k)}(t)=\varepsilon_{1}^{(k-1)}(t)-\lambda(t) \operatorname{Im}\left\langle\chi^{(k)}(t)\left|\left[\hat{H}_{1}+2 \hat{H}_{2} \varepsilon_{2}^{(k-1)}(t)\right]\right| \psi^{(k-1)}(t)\right\rangle
$$

and

$$
\frac{\partial}{\partial t} \psi^{(k)}(t)=-\left[\hat{H}_{0}+\frac{\hat{H}_{1}}{2}\left\{\varepsilon_{1}^{(k)}(t)+\varepsilon_{2}^{(k-1)}(t)\right\}+\hat{H}_{2} \varepsilon_{1}^{(k)}(t) \varepsilon_{2}^{(k-1)}(t)\right] \psi^{(k)}(t)
$$

with the initial condition, $\psi^{(k)}(0)=\psi_{0}$, and then $\varepsilon_{2}{ }^{(k)}(t)$ is updated as

$$
\varepsilon_{2}^{(k)}(t)=\varepsilon_{2}^{(k-1)}(t)-\lambda(t) \operatorname{Im}\left\langle\chi^{(k)}(t)\left|\left[\hat{H}_{1}+2 \hat{H}_{2} \varepsilon_{1}^{(k)}(t)\right]\right| \psi^{(k)}(t)\right\rangle .
$$

Here $\hat{H}_{0}$ is the field-free Hamiltonian and $\hat{H}_{1}$ and $\hat{H}_{2}$ are the molecular dipole moment and the polarizability, respectively, which are explicitly given below. The doubly divided electric-field components finally converge to the same value: $\lim _{k \rightarrow \infty} \varepsilon_{1}^{(k)}(t)=\lim _{k \rightarrow \infty} \varepsilon_{2}^{(k)}(t)=\varepsilon(t)$ because all the equations have a symmetric form with respect to $\varepsilon_{1}(t)$ and $\varepsilon_{2}(t)$.

\subsection{Wave-Packet Propagation}

We numerically solve the time-dependent Schrödinger equation:

$$
i \frac{\partial}{\partial t} \psi(t)=\hat{H} \psi(t)
$$

which describes the nuclear wave packet evolving with time. The total Hamiltonian $\hat{H}$ is the sum of the field-free and the field-molecule interaction parts, $\hat{H}_{0}+\hat{H}_{I}$. The field-free Hamiltonian $\hat{H}_{0}$ is composed of the kinetic energy operators for the radial distance $r$, the polar angle $\theta$, and the azimuth angle $\varphi$, and of the potential energy operator $V(r)$ :

$$
\hat{H}_{0}=-\frac{1}{2 m r^{2}}\left[\frac{\partial}{\partial r}\left(r^{2} \frac{\partial}{\partial r}\right)+\frac{1}{\sin \theta} \frac{\partial}{\partial \theta}\left(\sin \theta \frac{\partial}{\partial \theta}\right)+\frac{1}{\sin ^{2} \theta} \frac{\partial^{2}}{\partial \phi^{2}}\right]+V(r),
$$

where $m$ is the reduced mass. The calculated values for the $\mathrm{LiCl}$ ground $\mathrm{X}^{1} \Sigma^{+}$state potential [34] are used for $V(r)$. In the present study, the field-molecule interaction was considered non-perturbatively up to two-photon transitions and thus $\hat{H}_{I}$ is the sum of two terms $\varepsilon(t) \hat{H}_{1}$ and $\varepsilon^{2}(t) \hat{H}_{2}$ pertaining to oneand two-photon processes, respectively. Explicitly, $\hat{H}_{1}$ and $\hat{H}_{2}$ represent the molecular dipole moment and the polarizability, respectively:

$$
\begin{gathered}
\hat{H}_{1}=-\mu(r) \cos \theta, \\
\hat{H}_{2}=-\frac{1}{2}\left[\alpha_{\perp}+\left(\alpha_{\|}-\alpha_{\perp}\right) \cos ^{2} \theta\right],
\end{gathered}
$$

where $\alpha_{\perp}$ and $\alpha_{\|}$are the components of polarizability perpendicular and parallel, respectively, to the molecular axis. We employed the values of the dipole moment obtained in the previous work [34] and calculated the polarizability here using the Gaussian09 program [35] at the RMP2/aug-cc-pVTZ level of theory, Figure S1 in Supplementary Materials. It is known that the eigenfunction for $\hat{H}_{0}$ can be obtained by variable separation and the initial wave packet is set to an eigenfunction:

$$
\psi(0)=R_{v J}(r) Y_{J M_{J}}(\theta, \phi),
$$

where $Y_{J M_{J}}(\theta, \phi)$ is the spherical harmonics and $R_{v J}(r)$ is the $J$-dependent eigenfunction obtained by solving

$$
\left[-\frac{1}{2 m} \frac{\partial^{2}}{\partial r^{2}}+V(r)+\frac{1}{2 m r^{2}} J(J+1)\right] R_{V J}(r)=E_{\mho J} R_{V J}(r) .
$$


This is solved using the Fourier grid Hamiltonian method [36]. In the present calculations, only the $M_{J}$ $=0$ case is considered; this is justified because linearly polarized electric fields are used. Thus Equation (12) reduces to $\psi(0)=R_{v J}(r) \sqrt{(2 J+1) / 2} P_{J}^{0}(\cos \theta)$, where $P_{J}^{0}$ is the associated Legendre polynomials for $M_{J}=0$.

The system wave packet is represented as the complex amplitude at two-dimensional radial and angular grid points; radial grid points are chosen to be evenly spaced [36] and the angular ones as the Gauss-Legendre (GL) quadrature points [37]. The wave packet is evolved with time according to Equation (8), which is numerically integrated by using the split-operator method [38-40]:

$$
\begin{aligned}
\psi(t+\Delta t)=\exp & {\left[-i\left(\hat{T}_{R}+\hat{T}_{\theta}+V+\hat{H}_{I}\right) \Delta t\right] \psi(t) } \\
& \approx \exp \left[-i\left(V+\hat{H}_{I}\right) \Delta t / 2\right] \exp \left(-i \hat{T}_{\theta} \Delta t / 2\right) \exp \left(-i \hat{T}_{R} \Delta t\right) \\
& \times \exp \left(-i \hat{T}_{\theta} \Delta t / 2\right) \exp \left[-i\left(V+\hat{H}_{I}\right) \Delta t / 2\right] \psi(t)+O\left(\Delta t^{3}\right),
\end{aligned}
$$

where $\hat{T}_{R}$ and $\hat{T}_{\theta}$ are the radial and angular kinetic-energy operators, corresponding to the first and second terms, respectively, in Equation (9). A common trick to perform numerical actions of kinetic and potential-energy operators is to calculate each operator locally. The potential energy operator $V+\hat{H}_{I}$ is represented locally in coordinate space and its action on the wave packet is simply a multiplication. Since the kinetic-energy operators $\hat{T}_{R}$ and $\hat{T}_{\theta}$ are non-local in coordinate space, their operations on the wave packet are performed using the discrete variable representation (DVR) technique [41,42], as described below. When operating $\hat{T}_{R}$ on the wave packet, it is transformed from radial coordinate space into momentum counterpart with the forward fast-Fourier-transform (FFT) algorithm, being multiplied by the kinetic energy spectrum $k^{2} / 2 m$, and again it is transformed back into coordinate space with the reverse FFT algorithm. When operating $\hat{T}_{\theta}$, the wave packet in the point representation is transformed into the polynomial counterpart using the transformation matrix, $\mathbf{T}_{j i}=(j+1 / 2)^{1 / 2} w_{i}^{1 / 2} P_{j}^{0}\left(\sigma_{i}\right)$, where $\sigma_{i}=\cos \theta_{i}$ and $w_{i}$ are the points and weights for the GL quadrature; the transformed wave packet in the polynomial representation is multiplied by the spectrum $j(j+1) / 2 m r^{2}$, and again it is transformed back into the point representation with the reverse transformation matrix $\left(\mathbf{T}_{j i}\right)^{-1}$. To eliminate the artificial reflection of the wave packet at the edge of radial grid points, we incorporate a damping function [43] that is operative only for the last five points from the edge. Grid and time parameters used in the present OCT calculations are summarized in Table 1.

Table 1. Grid and time parameters used in the present OCT calculations.

\begin{tabular}{cc}
\hline Parameter & Value \\
\hline Range of the Li-Cl bond length (bohr) & $2.2677-5.6692$ \\
Number of radial grid points & 32 \\
Number of angular grid points & 20 \\
Total time (a.u.) & $2,000,000$ \\
Number of time steps & 131,072 \\
\hline
\end{tabular}

\section{Results and Discussion}

In the present OCT calculations, total time $T$ of the control pulse was set to 2,000,000 atomic units (a.u.) (48.4 ps), which is slightly larger than twice the rotational period of the $\mathrm{LiCl}$ molecule defined by the reciprocal of the $J=1 \leftarrow 0$ transition energy, $2 \pi / 2 B \sim 23 \mathrm{ps}$, where $B$ is the rotational constant and is $3.2 \times 10^{-6}$ a.u. for the ${ }^{7} \mathrm{Li}^{37} \mathrm{Cl}$ molecule. In each control excitation process we obtained weak and strong optimal fields by means of giving weak and strong field amplitudes, respectively, to the initial guess for the optimal field. 


\subsection{Rotational Excitation: $(v=0, J=0) \rightarrow(v=0, J=2)$}

\subsubsection{Weak Field}

The initial guess for the optimal field in the weak-field regime is

$$
\varepsilon(t)=E_{0} s(t)[\cos (2 B t)+\cos (4 B t)]
$$

with $E_{0}=1.0 \times 10^{-6}$ a.u. and $s(t)$ defined in Equation (3). This initial guess is employed assuming the one-photon resonant transition $J=1 \leftarrow 0$ followed by the transition $J=2 \leftarrow 1$. As summarized in Table 2 , the perfect control was achieved, i.e., the yield obtained by the optimal field is 1.000. The optimal field is very weak, as intended; the maximum field amplitude is $5.669 \times 10^{-6}$ a.u., and the fluence is $5.463 \times$ $10^{-6}$ a.u. Figure $1 \mathrm{a}$ shows the optimal field as a function of $t$ and Figure $1 \mathrm{~b}$ its spectrum. The spectrum is mostly composed of two frequency components centered around $\omega=6.0 \times 10^{-6}$ and $1.3 \times 10^{-5}$ a.u., which correspond to the initially guessed transition energies, $2 \mathrm{~B}$ and $4 \mathrm{~B}$, respectively.

Table 2. Properties of the resultant optimal electric fields.

\begin{tabular}{cccccc}
\hline & Init. Guess & Yield & $\begin{array}{c}\text { Max. Field } \\
\text { amp./a.u. }\end{array}$ & Fluence/a.u. & Filter/a.u. \\
\hline \multicolumn{7}{c}{ Rotational excitation: $(v=0, J=0) \rightarrow(v=0, J=2)$} \\
weak & Equation (15) & 1.000 & $5.669 \times 10^{-6}$ & $5.463 \times 10^{-6}$ & no filter \\
strong & Equation (18) & 0.950 & $7.505 \times 10^{-3}$ & 20.98 & $0<\omega<1.5 \times 10^{-4}$ \\
strong & Equation (19) & 0.959 & $1.991 \times 10^{-2}$ & 74.78 & $0<\omega<1.5 \times 10^{-4}$ \\
& \multicolumn{7}{c}{ Vibrational-rotational excitation: $(v=0, J=0) \rightarrow(v=1, J=2)$} & no filter \\
weak & Equation (20) & 0.997 & $7.200 \times 10^{-5}$ & $1.241 \times 10^{-3}$ & no filter \\
strong & Equation (21) & 0.998 & $1.014 \times 10^{-2}$ & 37.53 & no filter \\
strong & Equation (22) & 0.975 & $5.016 \times 10^{-2}$ & 469.06 & \\
\hline
\end{tabular}
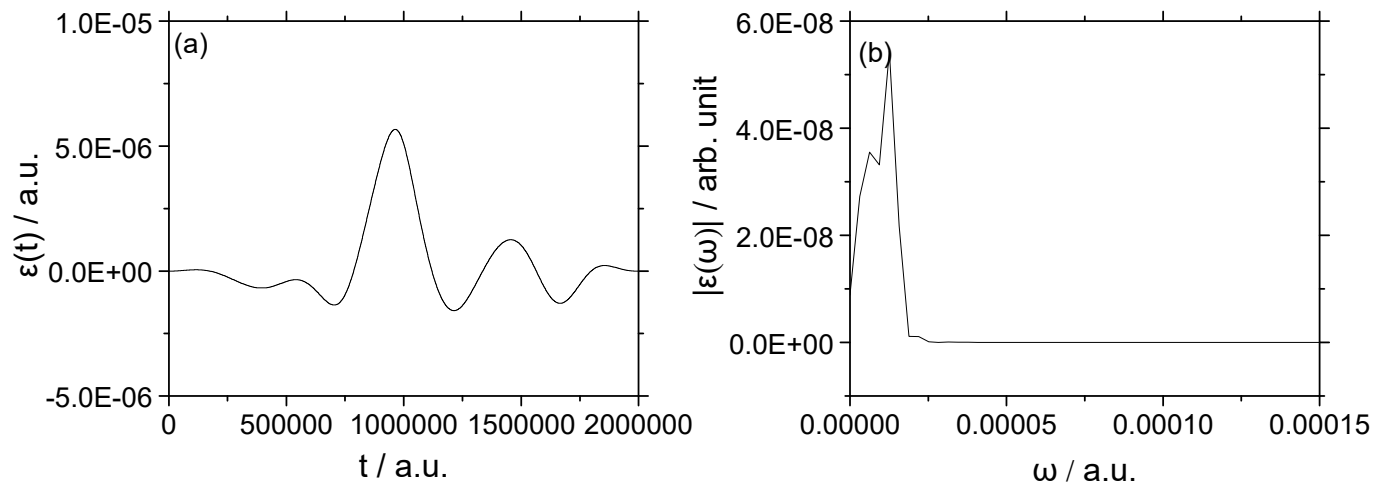

Figure 1. (a) The optimal field $\varepsilon(t)$ for the rotational excitation, $(v=0, J=0) \rightarrow(v=0, J=2)$ in the weak-field regime; (b) the spectrum of $\varepsilon(t)$.

Figure 2 shows temporal changes in the populations of rotational states. The time-dependent population of rotational state $J, p_{J}(t)$, is given here by the summation, over the number of radial grid point $N_{r}$, of the square of the projection of the rovibrational eigenfunction onto the system wave packet $\psi(t)$ :

$$
p_{J}(t)=\sum_{v}^{N_{r}}\left|\left\langle\psi(t) \mid R_{v J}(r) \sqrt{(2 J+1) / 2} P_{J}^{0}(\cos \theta)\right\rangle\right|^{2} .
$$


Similarly, that of vibrational state $v, p_{v}(t)$, is given by the summation, over the number of angular grid point $N_{a}$, of the same quantity:

$$
p_{v}(t)=\sum_{J}^{N_{a}}\left|\left\langle\psi(t) \mid R_{v J}(r) \sqrt{(2 J+1) / 2} P_{J}^{0}(\cos \theta)\right\rangle\right|^{2} .
$$

It was found that no vibrational excitation occurs in the present control process. As shown in Figure 2, the $J=1$ state is first excited, which is then followed by the $J=2$ state, and finally the $J=2$ state is totally populated at the end of the control pulse. It is seen that the $J=3$ state is slightly populated around the middle of the pulse.

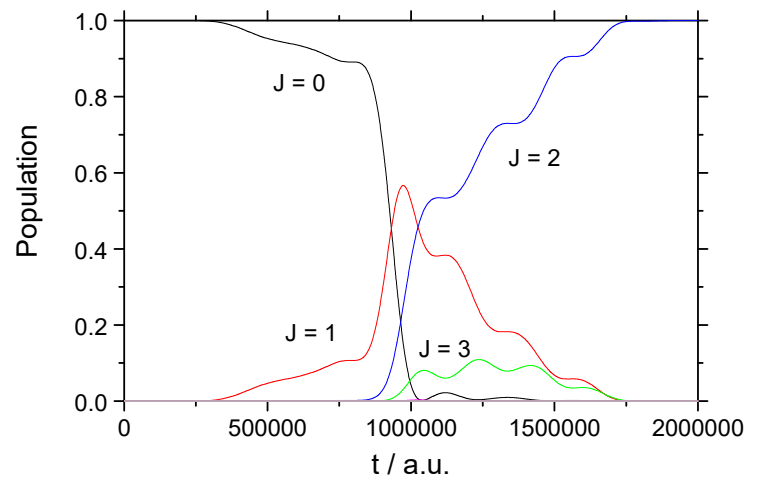

Figure 2. Temporal changes in state populations for the rotational excitation, $(v=0, J=0) \rightarrow(v=0, J=$ 2), in the weak-field regime.

It is thus concluded that the present rotational transition is controlled by only one-photon processes; the reasons are (i) the field intensity is very small; (ii) the field spectrum is composed of transition frequencies corresponding to the $J=1 \leftarrow 0$ and $J=2 \leftarrow 1$ transitions; (iii) the yield obtained for the partial Hamiltonian, $\hat{H}_{0}+\hat{H}_{1}$, with the optimal field was found to be the same as that for the full Hamiltonian, as summarized in Table 3.

Table 3. Comparison of the yields with the full $\left(\hat{H}_{0}+\hat{H}_{1}+\hat{H}_{2}\right)$ and partial Hamiltonians $\left(\hat{H}_{0}+\hat{H}_{1}\right.$ and $\hat{H}_{0}+\hat{H}_{2}$ ), obtained using the optimal fields for the full Hamiltonian.

\begin{tabular}{ccccc}
\hline & Init. Guess & $\hat{\mathbf{H}}_{\mathbf{0}}+\hat{\mathbf{H}}_{\mathbf{1}}+\hat{\mathbf{H}}_{\mathbf{2}}$ & $\hat{\mathbf{H}}_{\mathbf{0}}+\hat{\mathbf{H}}_{\mathbf{1}}$ & $\hat{\mathbf{H}}_{\mathbf{0}}+\hat{\mathbf{H}}_{\mathbf{2}}$ \\
\hline & Rotational excitation: $(v=0, J=0) \rightarrow(v=0, J=2)$ & \\
weak & Equation (15) & 1.000 & 1.000 & 0.000 \\
strong & Equation (18) & 0.950 & 0.184 & 0.338 \\
strong & Equation (19) & 0.959 & 0.009 & 0.633 \\
& Vibrational-rotational excitation: $(v=0, J=0) \rightarrow(v=1, J=2)$ & \\
weak & Equation (20) & 0.997 & 0.997 & 0.000 \\
strong & Equation (21) & 0.998 & 0.009 & 0.000 \\
strong & Equation (22) & 0.975 & 0.003 & 0.083 \\
\hline
\end{tabular}

\subsubsection{Strong Field}

In the strong-field regime we prepared two initial guesses for the optimal fields:

$$
\begin{gathered}
\varepsilon(t)=E_{0} s(t) \cos (3 B t) \\
\varepsilon(t)=E_{0} s(t)[\cos (6 B t)+\cos (12 B t)]
\end{gathered}
$$

with $E_{0}=7.5 \times 10^{-3}$ a.u. for both equations. Equations (18) and (19) are used assuming the two-photon absorption and Raman process, respectively. In these calculations frequency components higher than $1.5 \times 10^{-4}$ a.u. are filtered out in order to eliminate undesired high frequencies from the control field. 
As summarized in Table 2, the yield for the optimal field obtained with the initial guess, Equation (18), is 0.950 , the maximum field amplitude $7.505 \times 10^{-3}$ a.u., and the fluence 20.98 a.u. Figure 3a shows the optimal field as a function of $t$ and Figure $3 \mathrm{~b}$ its spectrum. The optimal field oscillates mostly with a single frequency, which is confirmed by the spectrum composed of a frequency component centered around $\omega=1.0 \times 10^{-5}$ a.u., corresponding to the initially guessed transition energy, 3B. As revealed in the inset in Figure 3b, however, frequency components with very small amplitudes are seen in the region $2.5 \times 10^{-5}<\omega<1.5 \times 10^{-4}$ a.u. When filtering out these components from the spectrum, we found it very difficult to achieve a high yield and they therefore contribute to the control mechanism significantly. Figure 4 shows temporal changes in the populations of (a) vibrational states and (b) rotational states. The populations of rotational states, Figure $4 \mathrm{~b}$, are highly oscillatory due to the high intensity of the control field and only the $J=2$ state is significantly populated at the end of the pulse. The vibrational states, Figure $4 \mathrm{a}$, other than $v=0$ are slightly populated and they are highly oscillatory also due to the high field intensity but the $v=0$ state is totally populated at the end of the pulse. Thus the intended control is achieved and the control mechanism is attributed mainly to a two-photon absorption because the field spectrum is mostly composed of a single frequency component, $\omega=$ 3B, corresponding to half the $J=2 \leftarrow 0$ transition energy, 6B. However, given the importance of other frequency components with small amplitudes, inset in Figure $3 b$, and the chaotic behaviors of the rotational state populations, Figure $4 \mathrm{~b}$, the control mechanism may be better characterized by a series of one- and two-photon processes, including not only two-photon absorption, but also the Raman process. As summarized in Table 3, the yields obtained for the partial Hamiltonians, $\hat{H}_{0}+\hat{H}_{1}$ and $\hat{H}_{0}+\hat{H}_{2}$, with the optimal field for the full Hamiltonian, are 0.184 and 0.338 , respectively, indicating that the one-photon process also contributes to the control mechanism. The yields obtained for the partial Hamiltonians suggest that the mechanism is nearly the simple addition of one-photon and two-photon processes.
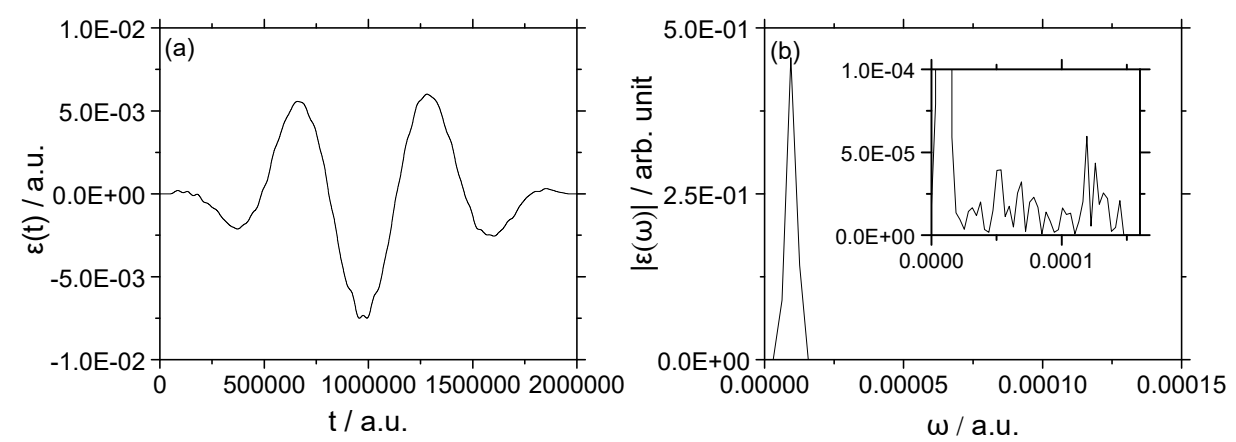

Figure 3. (a) The optimal field $\varepsilon(t)$ obtained with the initial guess, Equation (18), for the rotational excitation, $(v=0, J=0) \rightarrow(v=0, J=2)$, in the strong-field regime; (b) the spectrum of $\varepsilon(t)$.

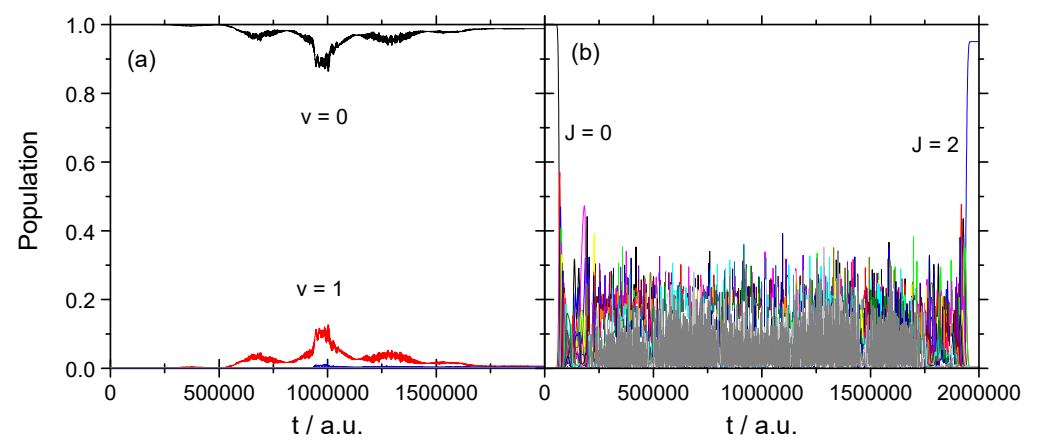

Figure 4. Temporal changes in state populations for the rotational excitation, $(v=0, J=0) \rightarrow(v=0$, $J=2$ ), caused by the optimal field shown in Figure 3, in the strong-field regime: (a) vibrational states; (b) rotational states. 
The yield for the optimal field obtained with the initial guess, Equation (19), is 0.959, the maximum field amplitude is $1.991 \times 10^{-2}$ a.u., and the fluence is 74.78 a.u. (Table 2). Figure 5a shows the optimal field as a function of $t$ and Figure $5 \mathrm{~b}$ its spectrum. The spectrum is mostly composed of two frequency components centered around $\omega=1.9 \times 10^{-5}$ and $3.8 \times 10^{-5}$ a.u., corresponding to the initially guessed transition energies, $6 \mathrm{~B}$ and $12 \mathrm{~B}$, respectively. The inset in Figure $5 \mathrm{~b}$ shows frequency components with very small amplitudes in the region $5.0 \times 10^{-5}<\omega<1.5 \times 10^{-4}$ a.u. We found it very difficult again to achieve a high yield without these components, suggesting that they contribute to the control mechanism significantly. It was found that temporal changes in the populations of vibrational and rotational states, Figure S2 in Supplementary Materials, are very similar to the last case with the initial guess, Equation (18), Figure 4; the behaviors of the rotational-state populations are chaotic and the vibrational states other than $v=0$ are slightly populated with high oscillation, thus the intended control being achieved at the end of the pulse. The control mechanism is attributed mainly to a Raman process because the field spectrum is mostly composed of two frequency components, $\omega=6 \mathrm{~B}$ and $12 \mathrm{~B}$, the difference of which corresponds to the $J=2 \leftarrow 0$ transition energy, 6B. Similarly to the above case, however, the control mechanism may be characterized by a series of one- and two-photon processes including both two-photon absorption and the Raman process. The yields obtained for the partial Hamiltonians, $\hat{H}_{0}+\hat{H}_{1}$ and $\hat{H}_{0}+\hat{H}_{2}$, with the optimal field for the full Hamiltonian, are 0.009 and 0.633 , respectively, Table 3 , suggesting that the two-photon process contributes more to the control mechanism than the other.
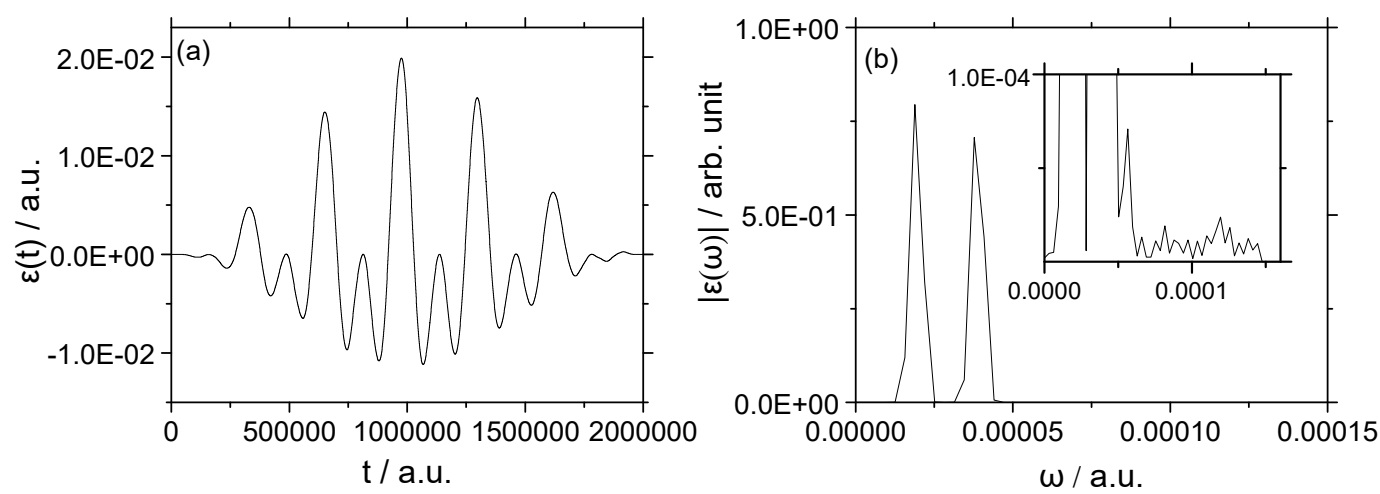

Figure 5. (a) The optimal field $\varepsilon(t)$ obtained with the initial guess, Equation (19), for the rotational excitation, $(v=0, J=0) \rightarrow(v=0, J=2)$, in the strong-field regime; (b) the spectrum of $\varepsilon(t)$.

3.2. Vibrational-Rotational Excitation: $(v=0, J=0) \rightarrow(v=1, J=2)$

\subsubsection{Weak Field}

In the weak-field regime the initial guess for the optimal field is

$$
\varepsilon(t)=E_{0} s(t) \cos \left(\omega_{01} t\right)
$$

with $E_{0}=1.0 \times 10^{-6}$ a.u. and $\omega_{01}$ being the $v=1 \leftarrow 0$ transition energy. As summarized in Table 2 , the achieved control is nearly perfect, i.e., the yield is 0.997 . As intended, the optimal field is very weak; the maximum field amplitude is $7.200 \times 10^{-5}$ a.u. and the fluence is $1.241 \times 10^{-3}$ a.u. Figure 6 a shows the optimal field as a function of $t$ and Figure $6 \mathrm{~b}$ its spectrum. The optimal field highly oscillates, causing the vibrational transition. The spectrum is mostly composed of a single frequency component centered around $\omega=3.0 \times 10^{-3}$ a.u.; closer inspection reveals that this exactly matches the energy of the $(v=0, J=0) \rightarrow(v=1, J=1)$ transition, $2.962 \times 10^{-3}$ a.u., which is an ordinary one-photon rovibrational transition. In order to achieve the targeted control, the additional rotational transition $J=2 \leftarrow 1$ is needed; the inset in Figure $6 \mathrm{~b}$ shows the close-up of the spectrum in the low frequency region, where there are some frequency components corresponding to rotational transitions, the largest of which, 
centered around $1.0 \times 10^{-5}$ a.u., almost matches the $J=2 \leftarrow 1$ transition energy, $4 \mathrm{~B}$. Figure 7 shows temporal changes in the populations of (a) vibrational states and (b) rotational states. The populations of vibrational states, Figure 7a, exhibit modest changes; the $v=0$ and 1 populations change slowly from 1 and 0 to 0 and 1, respectively. The rotational states, Figure $7 \mathrm{~b}$, also show modest changes but include high oscillations with small amplitudes and finally the $J=2$ population reaches 1 at the end.
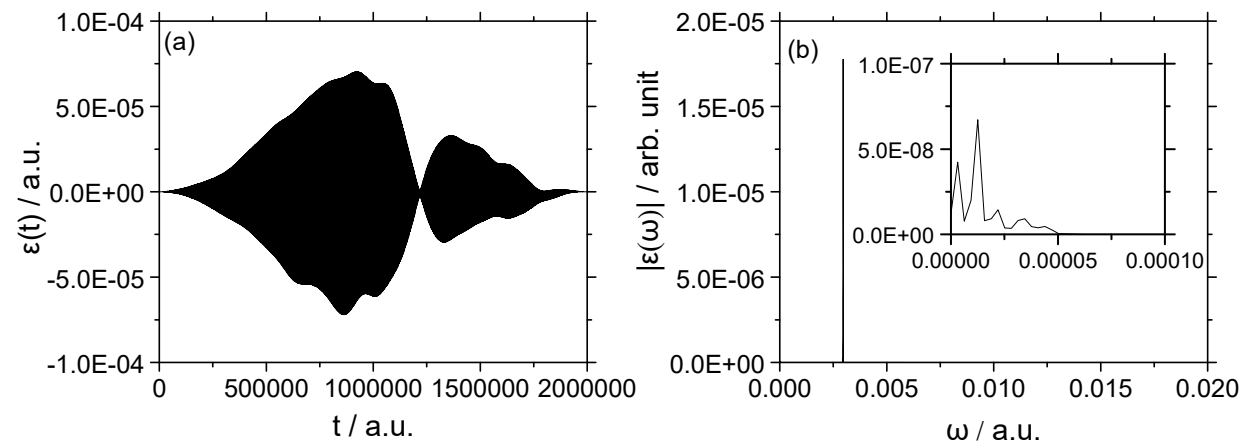

Figure 6. (a) The optimal field $\varepsilon(t)$ for the vibrational-rotational excitation, $(v=0, J=0) \rightarrow(v=1, J=2)$, in the weak-field regime; $(\mathbf{b})$ the spectrum of $\varepsilon(t)$.

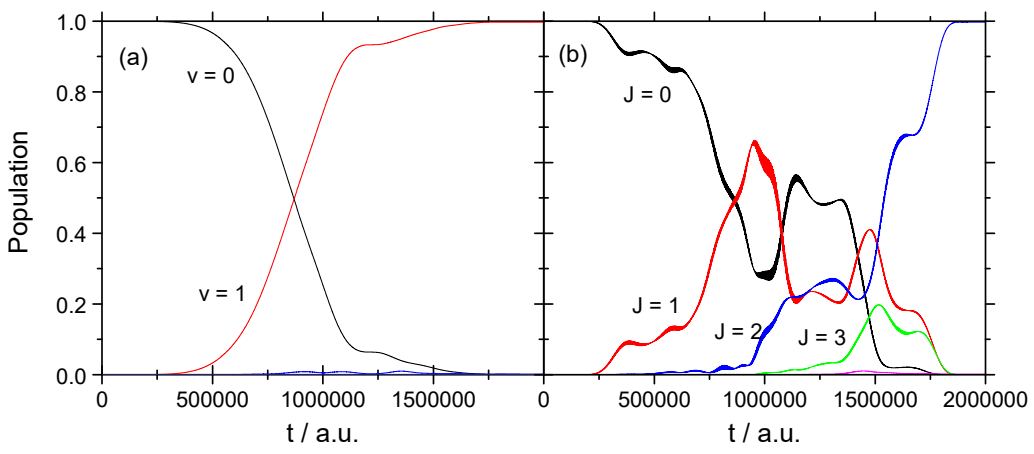

Figure 7. Temporal changes in state populations for the vibrational-rotational excitation, $(v=0, J=0)$ $\rightarrow(v=1, J=2)$, in the weak-field regime: (a) vibrational states; (b) rotational states.

To conclude, the present vibrational-rotational transition is controlled by only one-photon processes; the reasons are (i) the field intensity is very small; (ii) the field spectrum is composed of the frequencies of the $(v=0, J=0) \rightarrow(v=1, J=1)$ rovibrational transition and the $J=2 \leftarrow 1$ rotational transition; (iii) the yield obtained for the partial Hamiltonian, $\hat{H}_{0}+\hat{H}_{1}$, with the optimal field was found to be the same as that for the full Hamiltonian, as summarized in Table 3.

\subsubsection{Strong Field}

In the strong-field regime, we prepared two initial guesses again for the optimal fields:

$$
\begin{gathered}
\varepsilon(t)=E_{0} s(t) \cos \left(0.5 \omega_{01} t\right) \\
\varepsilon(t)=E_{0} s(t)\left[\cos \left(0.5 \omega_{01} t\right)+\cos \left(1.5 \omega_{01} t\right)\right]
\end{gathered}
$$

with $E_{0}=1.0 \times 10^{-2}$ a.u. for both equations. Equations (21) and (22) were used assuming the two-photon absorption and Raman process, respectively.

As summarized in Table 2, the yield for the optimal field obtained with the initial guess, Equation (21), is 0.998 , the maximum field amplitude is $1.014 \times 10^{-2}$ a.u., and the fluence is 37.53 a.u. Figure 8 a shows the optimal field as a function of $t$ and Figure $8 \mathrm{~b}$ its spectrum. The optimal field oscillates at a high frequency corresponding to the vibrational transition. The spectrum is mainly composed of a single frequency component centered around $\omega=1.5 \times 10^{-3}$ a.u., corresponding to the initially 
guessed transition energy, $0.5 \omega_{01}$. It thus follows that a two-photon absorption may play a key role in the control mechanism, as intended. The inset in Figure $8 \mathrm{~b}$ shows the close-up of the spectrum; in addition to the main frequency component around $\omega=1.5 \times 10^{-3}$ a.u., there are components of very small frequencies and the overtone of the main component. The small frequency components are associated with rotational transitions including very high rotational states, which is described below in temporal population analysis, and the overtone may contribute to the one-photon vibrational $v=$ $1 \leftarrow 0$ transition. Figure 9 shows temporal changes in the populations of (a) vibrational states and (b) rotational states. The vibrational states are excited up to around $v=6$ and the populations show very rapid changes with fast and slow oscillations. The $v=0$ and 1 populations, being 1 and 0 at $t=0$, finally reach 0 and 1 , respectively, at the end of the pulse. The rotational state populations show extremely fast and sharp oscillations due to the high intensity of the control field and the states up to nearly $J=$ 20 are populated, but only the $J=2$ state is highly populated at the end of the pulse. Thus the targeted control is achieved and the mechanism is likely to be dominated by a two-photon absorption because the field spectrum is composed of a single frequency component, $0.5 \omega_{01}$, corresponding to half the $v=$ $1 \leftarrow 0$ transition energy. However, many frequencies other than the main component, inset in Figure 8b, and the chaotic behaviors of the vibrational and rotational state populations, Figure 9, suggest that the control mechanism is characterized by multiple one- and two-photon processes including both two-photon absorption and the Raman process. Table 3 shows that the yields obtained for the partial Hamiltonians, $\hat{H}_{0}+\hat{H}_{1}$ and $\hat{H}_{0}+\hat{H}_{2}$, with the optimal field, are 0.009 and 0.000 , respectively, indicating that neither the one- and two-photon processes work independently. Rather, they have a kind of cooperative effect of one- and two-photon processes, which is in contrast to the control mechanisms for the rotational excitation, $(v=0, J=0) \rightarrow(v=0, J=2)$, with the strong fields, where one- and two-photon processes are distinct to some extent and the cooperative effect is small, Table 3.
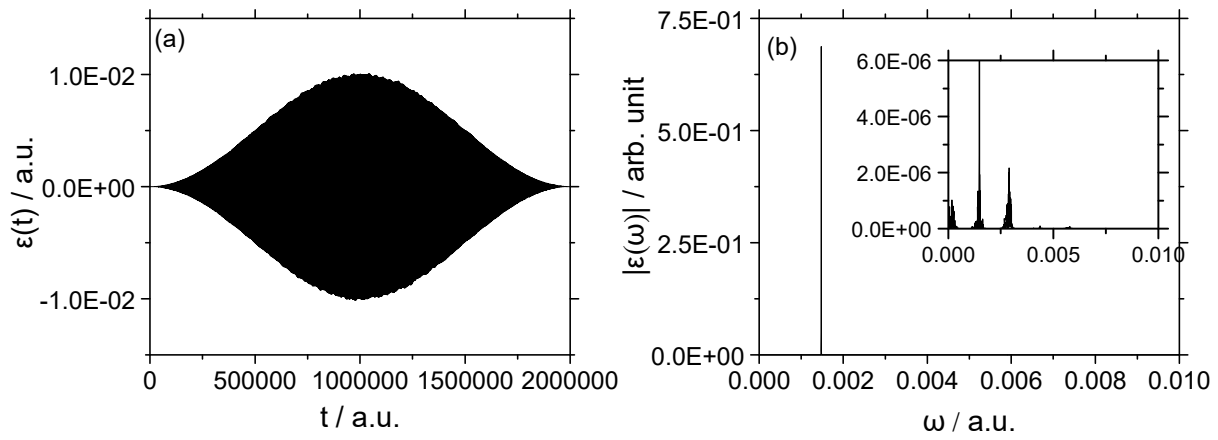

Figure 8. (a) The optimal field $\varepsilon(t)$ obtained with the initial guess, Equation (21), for the vibrational-rotational excitation, $(v=0, J=0) \rightarrow(v=1, J=2)$, in the strong-field regime; (b) the spectrum of $\varepsilon(t)$.

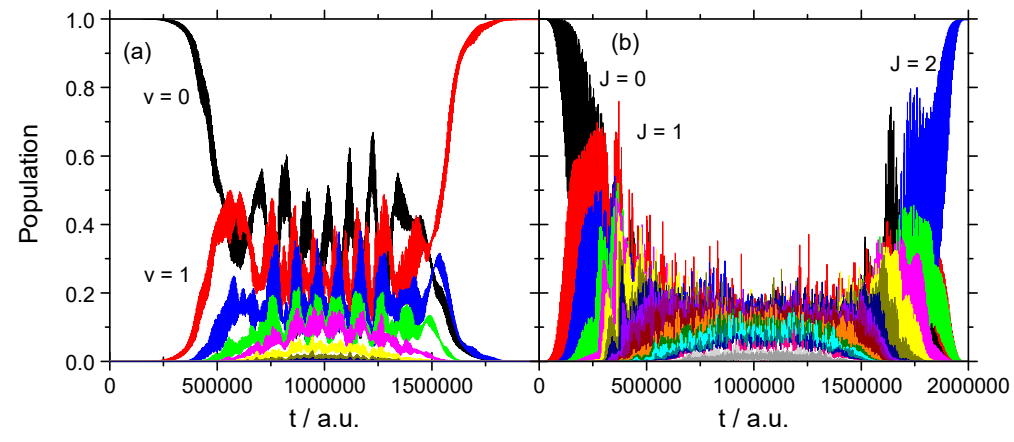

Figure 9. Temporal changes in state populations for the vibrational-rotational excitation, $(v=0, J=0)$ $\rightarrow(v=1, J=2)$, caused by the optimal field shown in Figure 8, in the strong-field regime: (a) vibrational states; (b) rotational states. 
The optimal field obtained using the initial guess, Equation (22), is found to give the final yield, 0.975 , with its maximum field amplitude being $5.016 \times 10^{-2}$ a.u. and its fluence being 469.06 a.u., Table 2. Figure 10a shows the optimal field as a function of $t$ and Figure $10 \mathrm{~b}$ its spectrum. The optimal field oscillates at a high frequency corresponding to the vibrational transition. The spectrum is mainly composed of two frequency components centered around $\omega=1.5 \times 10^{-3}$ and $3.0 \times 10^{-3}$ a.u., corresponding to the initially guessed transition energy. Hence a Raman process may play a key role in the control mechanism, as intended. The close-up of the spectrum is shown in the inset in Figure 10b; other than the two main frequency components, there are components with small amplitudes corresponding to rotational transitions and the $v=1 \leftarrow 0$ vibrational transition. Temporal changes in the populations of vibrational and rotational states, Figure S3 in Supplementary Materials, are found to show similar behavior to those obtained with the initial guess, Equation (21), Figure 9; the vibrational state populations oscillate quite rapidly and so do the rotational state populations, the $v=1$ and $J=2$ states being highly populated at the end of the pulse. Thus the mechanism is likely due to a Raman process because the field spectrum is composed of two frequency components, $0.5 \omega_{01}$ and $1.5 \omega_{01}$, corresponding to absorption and emission via a virtual state located at $1.5 \omega_{01}$. As with the above case, however, the control mechanism may be better characterized by multiple oneand two-photon processes rather than a simple Raman process. The yields obtained for the partial Hamiltonians, $\hat{H}_{0}+\hat{H}_{1}$ and $\hat{H}_{0}+\hat{H}_{2}$, with the optimal field, are 0.003 and 0.083 , respectively, as given in Table 3, showing that the cooperative effect is very large like the last result.
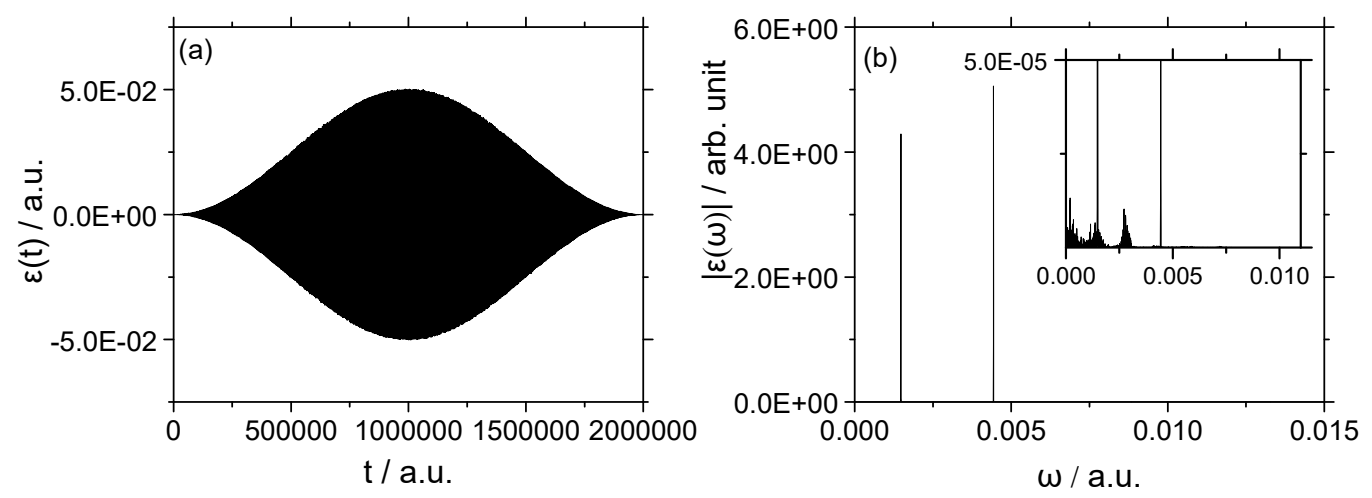

Figure 10. (a) The optimal field $\varepsilon(t)$ obtained with the initial guess, Equation (22), for the vibrational-rotational excitation, $(v=0, J=0) \rightarrow(v=1, J=2)$, in the strong-field regime; (b) the spectrum of $\varepsilon(t)$.

\section{Conclusions}

In this work we investigated the roles of one-photon and two-photon processes in the laser-controlled rovibrational transitions of the diatomic alkali halide, ${ }^{7} \mathrm{Li}^{37} \mathrm{Cl}$. We carried out OCT calculations to obtain the electric fields that best yield the transition probability to the target. The Hamiltonian including both the one-photon and two-photon field-molecule interaction terms was used and the 2D time-dependent wave packet propagation was performed with both the radial and angular motions being treated quantum mechanically. The targeted processes were pure rotational and vibrational-rotational excitations: $(v=0, J=0) \rightarrow(v=0, J=2) ;(v=0, J=0) \rightarrow(v=1, J=2)$. Total time of the control pulse was set to 2,000,000 a.u. (48.4 ps), which is slightly larger than twice the rotational period of the ${ }^{7} \mathrm{Li}^{37} \mathrm{Cl}$ molecule (23 ps). In each control excitation process we obtained weak and strong optimal fields by means of giving weak and strong field amplitudes, respectively, to the initial guess for the optimal field.

In the weak-field regime it was found that the control mechanism is dominated exclusively by a one-photon process, as expected, in both the rotational and vibrational-rotational transitions. In the strong-field regime we obtained two kinds of optimal fields: one that mainly causes two-photon absorption and the other a Raman process. It was found, however, that the mechanisms are not simply 
characterized by one process but rather by multiple one- and two-photon processes including both two-photon absorption and the Raman process. In the rotational excitation, $(v=0, J=0) \rightarrow(v=$ $0, J=2)$, it was found that the roles of one- and two-photon processes are relatively distinct; in the vibrational-rotational excitation, $(v=0, J=0) \rightarrow(v=1, J=2)$, however, the roles of the two processes are ambiguous and the cooperative effect is quite large. We thus obtained the various optimal fields to efficiently achieve the target controlled by different mechanisms depending on the field strength and the type of quantum transition.

Supplementary Materials: Supplementary data to this article can be found online at http://www.mdpi.com/22181997/5/5/109/s1.

Author Contributions: Both the authors conceived of the simulations; Y.K. designed and performed the OCT calculations and K.Y. reviewed the data; Y.K. prepared the original paper draft and K.Y. reviewed and edited.

Funding: This work was supported by JSPS KAKENHI Grant No. 15H02345.

Acknowledgments: The authors thank Yukiyoshi Ohtsuki at Tohoku University for helpful suggestions. The computations were performed using the supercomputers at Center for Computational Science \& e-Systems, JAEA, Tokai, Ibaraki, Japan and also at Research Center for Computational Science, Okazaki, Aichi, Japan.

Conflicts of Interest: The authors declare no conflict of interest.

\section{References}

1. Rice, S.A.; Zhao, M. Optical Control of Molecular Dynamics; Wiley: New York, NY, USA, 2000.

2. Letokhov, V.S. Laser Control of Atoms and Molecules; Oxford University Press: New York, NY, USA, 2007.

3. D'alessandro, D. Introduction to Quantum Control and Dynamics; Chapman \& Hall: London, UK, 2007.

4. Shapiro, M.; Brumer, P. Quantum Control of Molecular Processes; Wiley-VCH: Weinheim, Germany, 2012.

5. Kosloff, R.; Rice, S.A.; Gaspard, P.; Tersigni, S.; Tannor, D.J. Wavepacket dancing: Achieving chemical selectivity by shaping light pulses. Chem. Phys. 1989, 139, 201-220. [CrossRef]

6. Brumer, P.; Shapiro, M. Coherence chemistry: Controlling chemical reactions with lasers. Acc. Chem. Res. 1989, 22, 407-413. [CrossRef]

7. Stapelfeldt, H.; Seideman, T. Aligning molecules with strong laser pulses. Rev. Mod. Phys. 2003, 75, 543. [CrossRef]

8. Nielsen, M.A.; Chuang, I.L. Quantum Computation and Quantum Information; Cambridge University Press: Cambridge, UK, 2000.

9. Bartana, A.; Kosloff, R.; Tannor, D.J. Laser cooling of molecular internal degrees of freedom by a series of shaped pulses. J. Chem. Phys. 1993, 99, 196-210. [CrossRef]

10. McCarron, D.J.; Steinecker, M.H.; Zhu, Y.; DeMille, D. Magnetic trapping of an ultracold gas of polar molecules. Phys. Rev. Lett. 2018, 121, 013202. [CrossRef] [PubMed]

11. Bergmann, K.; Theuer, H.; Shore, B.W. Coherent population transfer among quantum states of atoms and molecules. Rev. Mod. Phys. 1998, 70, 1003. [CrossRef]

12. Vitanov, N.V.; Rangelov, A.A.; Shore, B.W.; Bergmann, K. Stimulated Raman adiabatic passage in physics, chemistry, and beyond. Rev. Mod. Phys. 2017, 89, 015006. [CrossRef]

13. Falci, G.; Di Stefano, P.G.; Ridolfo, A.; D'Arrigo, A.; Paraoanu, G.S.; Paladino, E. Advances in quantum control of three-level superconducting circuit architectures. Prog. Phys. 2017, 65, 1600077. [CrossRef]

14. Torrontegui, E.; Ibánez, S.; Martínez-Garaot, S.; Modugno, M.; del Campo, A.; Guéry-Odelin, D.; Ruschhaupt, A.; Chen, X.; Muga, J.G. Shortcuts to adiabaticity. Adv. At. Mol. Opt. Phys. 2013, 62, 117-169.

15. Vepsäläinen, A.; Danilin, S.; Paraoanu, G.S. Optimal superadiabatic population transfer and gates by dynamical phase corrections. Quantum Sci. Technol. 2018, 3, 024006. [CrossRef]

16. Vepsäläinen, A.; Danilin, S.; Paraoanu, G.S. Superadiabatic population transfer in a three-level superconducting circuit. Sci. Adv. 2019, 5, eaau5999. [CrossRef]

17. Shi, S.; Rabitz, H. Quantum mechanical optimal control of physical observables in microsystems. J. Chem. Phys. 1990, 92, 364-376. [CrossRef]

18. Zhu, W.; Botina, J.; Rabitz, H. Rapidly convergent iteration methods for quantum optimal control of population. J. Chem. Phys. 1998, 108, 1953-1963. [CrossRef] 
19. Sundermann, K.; de Vivie-Riedle, R. Extensions to quantum optimal control algorithms and applications to special problems in state selective molecular dynamics. J. Chem. Phys. 1999, 110, 1896-1904. [CrossRef]

20. Levis, R.; Rabitz, H. Closing the loop on bond selective chemistry using tailored strong field laser pulses. J. Phys. Chem. A 2002, 106, 6427-6444. [CrossRef]

21. Herek, J.L.; Wohllenben, W.; Cogdell, R.J.; Zeldler, D.; Motzkus, M. Quantum control of energy flow in light harvesting. Nature 2002, 417, 533-535. [CrossRef]

22. Kurosaki, Y.; Yokoyama, K.; Yokoyama, A. Multilevel effect on ultrafast isotope-selective vibrational excitations: Quantum optimal control study. J. Mol. Struct. Theochem. 2009, 913, 38-42. [CrossRef]

23. Kurosaki, Y.; Yokoyama, K.; Yokoyama, A. Quantum control study of multilevel effect on ultrafast isotope-selective vibrational excitations. J. Chem. Phys. 2009, 131, 144305. [CrossRef]

24. Kurosaki, Y.; Yokoyama, K.; Yokoyama, A. Quantum control study of ultrafast isotope-selective vibrational excitations of the cesium iodide (CsI) molecule. Comput. Theoret. Chem. 2011, 963, 245-255. [CrossRef]

25. Kurosaki, Y.; Ichihara, A.; Yokoyama, K. Quantum optimal control for the full ensemble of randomly oriented molecules having different field-free Hamiltonians. J. Chem. Phys. 2011, 135, 054103. [CrossRef]

26. Yokoyama, K.; Matsuoka, L.; Kasajima, T.; Tsubouchi, M.; Yokoyama, A. Quantum control of molecular vibration and rotation toward the isotope separation. In Advances in Intense Laser Science and Photonics; Lee, J., Ed.; Publishing House for Science and Technology: Hanoi, Vietnam, 2010; p. 113.

27. Matsuoka, L.; Ichihara, A.; Hashimoto, M.; Yokoyama, K. Theoretical study for laser isotope separation of heavy-element molecules in a thermal distribution. In Proceedings of the International Conference on toward and over the Fukushima Daiichi Accident (GLOBAL 2011), Tokyo, Japan, 11-16 December 2011.

28. Ichihara, A.; Matsuoka, L.; Kurosaki, Y.; Yokoyama, K. Theoretical study on isotope-selective dissociation of the lithium chloride molecule using a designed terahertz-wave field. In Proceedings of the 12th Asia Pacific Physics Conference (APPC12), Chiba, Japan, 14-19 July 2013; p. 013093.

29. Ichihara, A.; Matsuoka, L.; Kurosaki, Y.; Yokoyama, K. An analytic formula to describe transient rotational dynamics of diatomic molecules in an optical frequency comb. Chin. J. Phys. 2013, 51, 1230-1340.

30. Ichihara, A.; Matsuoka, L.; Kurosaki, Y.; Yokoyama, K. Quantum control of isotope-selective rovibrational excitation of diatomic molecules in the thermal distribution. Opt. Rev. 2015, 22, 153-156. [CrossRef]

31. Kurosaki, Y.; Yokoyama, K. Quantum optimal control of the isotope-selective rovibrational excitation of diatomic molecules. Chem. Phys. 2017, 493, 183-193. [CrossRef]

32. Ohtsuki, Y.; Nakagami, K. Monotonically convergent algorithms for solving quantum optimal control problems of a dynamical system nonlinearly interacting with a control. Phys. Rev. A 2008, 77, 033414. [CrossRef]

33. Abe, H.; Ohtsuki, Y. Frequency-network mechanism for alignment of diatomic molecules by multipulse excitation. Phys. Rev. A 2011, 83, 053410. [CrossRef]

34. Kurosaki, Y.; Yokoyama, K. Ab initio MRSDCI study on the low-lying electronic states of the lithium chloride molecule (LiCl). J. Chem. Phys. 2012, 137, 064305. [CrossRef]

35. Frisch, M.J.; Trucks, G.W.; Schlegel, H.B.; Scuseria, G.E.; Robb, M.A.; Cheeseman, J.R.; Scalmani, G.; Barone, V.; Mennucci, B.; Petersson, G.A.; Revision, D.; et al. Gaussian 09; Revision D.01; Gaussian, Inc.: Wallingford, CT, USA, 2013.

36. Marston, C.C.; Balint-Kurti, G.G. The Fourier grid Hamiltonian method for bound state eigenvalues and eigenfunctions. J. Chem. Phys. 1989, 91, 3571-3576. [CrossRef]

37. Press, W.H.; Flannery, B.P.; Teukolsky, S.A.; Vetterling, T. Numerical Recipes; Cambridge University Press: Cambridge, MA, USA, 1986.

38. Feit, M.D.; Fleck, J.A., Jr.; Steiger, A. Solution of the Schrödinger equation by a spectral method. J. Comput. Phys. 1982, 47, 412-433. [CrossRef]

39. Feit, M.D.; Fleck, J.A., Jr. Solution of the Schrödinger equation by a spectral method II: Vibrational energy levels of triatomic molecules. J. Chem. Phys. 1983, 78, 301-308. [CrossRef]

40. Feit, M.D.; Fleck, J.A., Jr. Wave packet dynamics and chaos in the Hénon-Heiles system. J. Chem. Phys. 1984, 80, 2578-2584. [CrossRef]

41. Light, J.C.; Hamilton, I.P.; Lill, V.J. Generalized discrete variable approximation in quantum mechanics. J. Chem. Phys. 1985, 82, 1400-1409. [CrossRef] 
42. Dai, J.; Light, J.C. A grid representation for spherical angles: Decoupling of the angular momentum operator. J. Chem. Phys. 1997, 107, 8432-8436. [CrossRef]

43. Mahapatra, S.; Sathyamurthy, N. Negative imaginary potentials in time-dependent quantum molecular scattering. J. Chem. Soc. Faraday Trans. 1997, 93, 773-779. [CrossRef]

(C) 2019 by the authors. Licensee MDPI, Basel, Switzerland. This article is an open access article distributed under the terms and conditions of the Creative Commons Attribution (CC BY) license (http://creativecommons.org/licenses/by/4.0/). 